\title{
Moving Risk of Crowds in the Entrance Confluence Area in the Presence of Channelizing Facilities
}

\author{
Yifan Zhuang ${ }^{1} \cdot$ Zhigang Liu' $^{1}$. Jiajun Huang ${ }^{2}$. Lizhong Yang ${ }^{3}$. \\ Andreas Schadschneider 4 \\ ${ }^{1}$ College of Urban Railway Transportation, Shanghai University of Engineering Science, \\ Shanghai, China \\ E-mail: zhuangyf@sues.edu.cn, Izg@sues.edu.cn \\ 2 Shanghai Electric Automation Group, Shanghai, China \\ E-mail: 243605334@qq.com \\ ${ }^{3}$ State Key Laboratory of Fire Science, University of Science and Technology of China, Hefei, \\ China \\ E-mail: yanglz@ustc.edu.cn \\ 4 Institute for Theoretical Physics, University of Cologne, Köln, Germany \\ E-mail: as@thp.uni-koeln.de
}

Received: 23 August 2021 / Last revision received: 6 November 2021 / Accepted: 11 November 2021 DOI: $10.17815 / \mathrm{CD} .2021 .130$

\begin{abstract}
In recent years, the measures to interfere the crowds movement with physical facilities (such as channelizing, separation railing) have become more and more common, but how they affect the crowd movement and what moving risks exist in the entrance confluence area have not been fully revealed. Therefore, this paper analyzes the moving risk of the crowds before the bottleneck entrance area, in the presence of the channelizing barriers by controllable laboratory experiments. The visual color cloud charts of the local density, speed and confusion degree of moving directions within the entrance confluence area are analyzed in the presence of different gaps $(1.05 \mathrm{~m}$ and $0.7 \mathrm{~m})$ channelizing barriers, to further quantify the motion risk of the crowds. The study finds that the narrower gaps of the channelizing railings, the larger area of high-risk zones, and they could have clear 'lane formation' effect in shaping the risk zones. The both ends of the channelizing barriers are higher moving risk zones for multi-entry sides conditions, but the area before the middle channels also needs to be closely concerned when the participants enter from two opposite entering sides. The study will provide theoretical basis for identify hotspots and danger zones in the entrance confluence area, which is helpful for the design of the channelizing barriers and conducting scientific crowd management decisions.
\end{abstract}


Keywords Channelizing facilities $\cdot$ crowds management $\cdot$ experiments $\cdot$ risk visualization $\cdot$ bottleneck $\cdot$ entrance

\section{Introduction}

There are always highly gathered pedestrians around the entrances of the bottlenecks (e.g. pedestrian facilities, channel entrance, etc.) in public places. Promoting the safety, rapid and orderly traffic of bottleneck entrances, is an important prerequisite for preventing the gathering risk of crowds. Therefore, the implementation of effective intervention strategies for the dense population at the entrance of the bottleneck is the top priority of the safety management of public places. Among them, the physical intervention strategy is to restrict the flow of the crowd by adding physical barriers such as railings and obstacles in front of the bottleneck entrance to achieve the purpose of adjusting the temporal and spatial distribution of crowd movement, including the arrangement of serpentine lines [1], the placement of channelized railings/iron horses [2,3], the placement of obstacles of different shapes [4], and a combination of the above methods. However, physical intervention measures separate and restrict the movement space of pedestrians at the bottleneck, which may cause certain interference to crowd movement. In addition, complex crowd movements such as confluence and multi-directional flow are likely to cause delays and queuing phenomena at the entrance of the bottleneck [5,6], which increases the risk of jamming and conflicts [7, 8]. Many researchers applied the crowd density [9], the fundamental diagram [10], the Level of Service (LOS) [11] or local velocity [12] etc. to evaluate the moving risk of the pedestrian crowds, but most of their estimations are limited by the sensing methods, geometries and different density or flow conditions [8]. It's vital to develop an universal implementation to understand the moving risk in the frame of crowd management. Therefore, it is necessary to evaluate and test the movement risk of people from different directions when physical interventions are installed, so as to understand the impact of such interventions on the movement characteristics of people from a safety perspective.

This article mainly focuses on the characteristics of multi-directional crowd movement in the confluence area at the entrance of the aisle in the presence of channelizing railings. A visual method for risk assessment that considers the movement of the crowd in the whole process is proposed. It can comprehensively reveal the temporal and spatial distribution of local density, speed, directional chaos and other movement characteristic parameters, and it can also directly reflect the risk distribution situation in the entrance confluence area. The results can provide a theoretical basis for crowd managers to take appropriate physical intervention measures.

\section{Experiment Settings}

These experiments were conducted in 2018 at a university in Hefei, China. A total of 126 people, 80 male and 46 female, participated in the experiments, all of whom were 
college students with an average height of $1.70 \mathrm{~m}( \pm 0.08 \mathrm{~m})$ and an average age of 20 years old. We employed an indoor positioning technology Ultra-wideband (UWB) to automatically record and display the real movement trajectories, which calculates the time difference of arrival (TDOA) of the signals to obtain the distance between the reference point and the target [13]. The advantages of UWB are high accuracy, being unaffected by interference, and effectively passing through walls and obstacles, so it is used for various indoor positioning and tracking applications in hospitals, shopping malls, airports, museums, athlete training and others $[14,15]$. In these experiments, four base stations were fixed atop 4 supports in $3.0 \mathrm{~m}$ high at four outside corners in Fig. 1(a), receiving signals emitted from active electronic tags affixed to the headgear of each participant (see Fig. 1(b)). The UWB system can obtain 8000 coordinates per second, and its positioning precision is $0.01-0.1 \mathrm{~m}$.

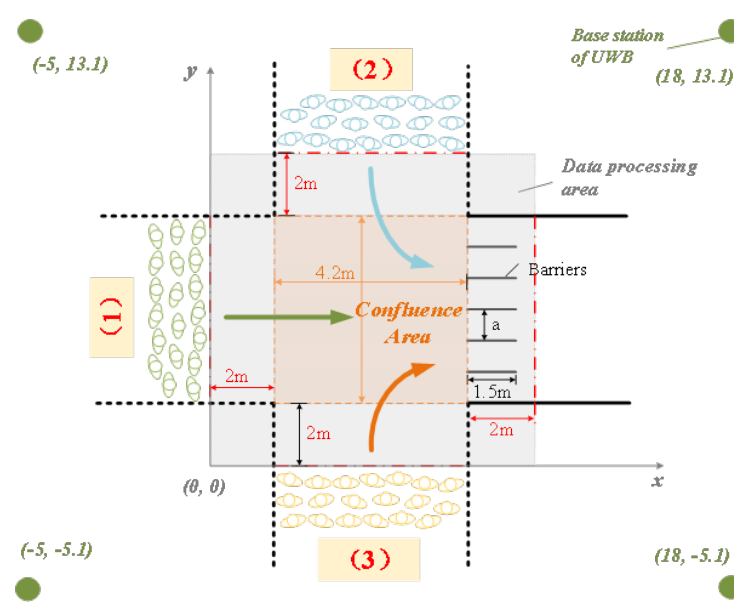

(a)

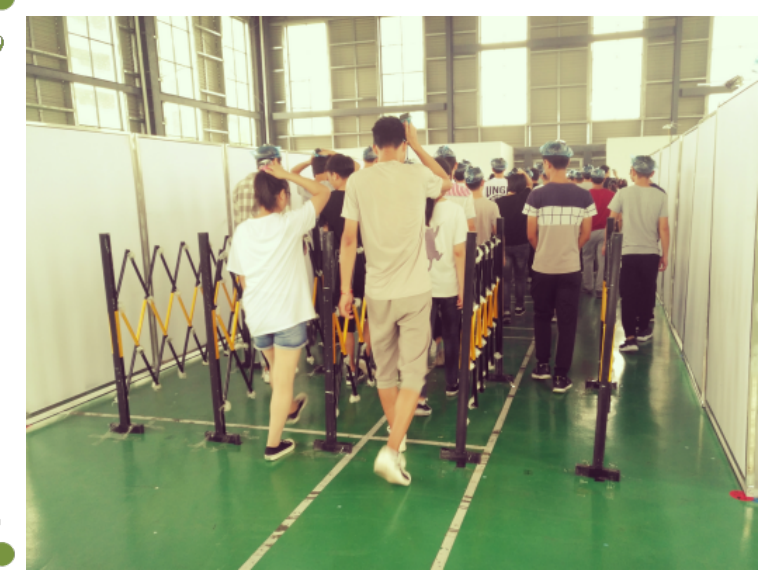

(b)

Figure 1 (a) The scene of evacuation experiment. (b) Photographs at the entrance of channelizing facilities.

As shown in Fig. 1, the participants were required to stand evenly in the waiting area (1) - (3), two meters away from the confluence area (a square with sides as $4.2 \mathrm{~m}$ ) in the front of the aisle. The channelizing facilities were barriers side by side at the entrance of the aisle, and their lengths were $1.5 \mathrm{~m}$. But their gaps $a$ were changed as $0.7 \mathrm{~m}$ and $1.05 \mathrm{~m}$ in different experiment scenes, which could admit the participants to walk alone and at most two abreast, respectively. When the experiments started, the volunteers moved across the confluence area and passed through the channelizing facilities at normal speed. All their position coordinates and the corresponding time were processed and analyzed within the gray square area (Fig. 1(a)). In this experiment, eight conditions were conducted as in Tab. 1: 


\begin{tabular}{lllll}
\hline $\begin{array}{l}\text { Serial } \\
\text { num- } \\
\text { ber }\end{array}$ & Entering area & $\begin{array}{l}\text { Number of } \\
\text { each enter- } \\
\text { ing area }\end{array}$ & Aap $a$ & \\
\hline 1 & waiting area $(1)$ & 126 people & $0.7 \mathrm{~m}$ & $W(1)-a 0.7$ \\
2 & waiting area $(2)$ & 126 people & $0.7 \mathrm{~m}$ & $W(2)-a 0.7$ \\
3 & waiting area $(2),(3)$ & 63 people & $0.7 \mathrm{~m}$ & $W(2 / 3)-a 0.7$ \\
4 & waiting area $(1),(2),(3)$ & 42 people & $0.7 \mathrm{~m}$ & $W(1 / 2 / 3)-a 0.7$ \\
5 & waiting area $(1)$ & 126 people & $1.05 \mathrm{~m}$ & $W(1)-a 1.05$ \\
6 & waiting area $(2)$ & 126 people & $1.05 \mathrm{~m}$ & $W(2)-a 1.05$ \\
7 & waiting area $(2),(3)$ & 63 people & $1.05 \mathrm{~m}$ & $W(2 / 3)-a 1.05$ \\
8 & waiting area $(1),(2),(3)$ & 42 people & $1.05 \mathrm{~m}$ & $W(1 / 2 / 3)-a 1.05$ \\
\hline
\end{tabular}

Table 1 Eight experiment conditions.

\section{Risk visualization calculation method}

Based on this data extracted by UWB, a visualization calculation method is proposed for demonstrating the moving risk situation of the data processing area (Fig. 1(b)). Twodimensional square cells are employed to subdivide the area and the size of each cell is $0.4 \times 0.4 \mathrm{~m}^{2}$ (also can be smaller size), which allows to obtain accurate results and ensure an adequate space and spatial resolution. The local density, speed and confusion degree of moving directions at cell $(i, j)$ are measured in the calculation region (Fig. 2).

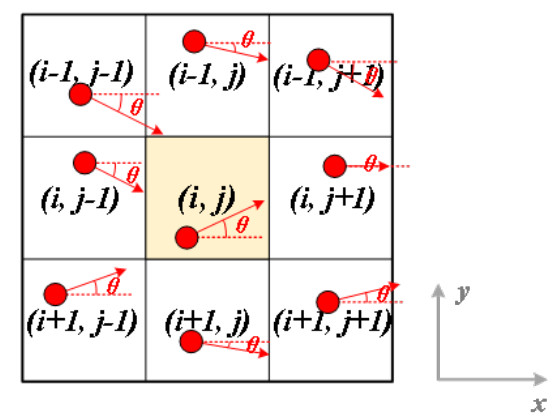

Figure 2 Calculation region of cell $(i, j)$, which contains the cell $(i, j)$ and its Moore neighborhood cells. Red circles represent the persons in the calculation region at time $t$.

The local density $L_{d}(t)$ at cell $(i, j)$ is calculated by Eq. 1 :

$$
L_{d}(t)=\frac{N(t)}{A_{\text {region }}}
$$

where $N(t)$ is the number of people occupied in the the calculation region (nine cells) in Fig. 2 at time $t$. A region is the area of the calculation region, and the unit is $m^{2}$. The local speed $L_{S}(t)$ at cell $(i, j)$ is calculated by Eq. 2 : 


$$
L_{s}(t)=\frac{\sum_{\text {region }} v(t)}{N(t)}
$$

where $L_{s}(t)$ is the mean value of the instantaneous velocities $v(t)$ for the whole persons $N(t)$ in the calculation region at time $t$, and the unit is $\mathrm{m} / \mathrm{s}$. The local confusion degree of moving directions $L_{c d}(t)$ at cell $(i, j)$ is calculated by Eq. 3 :

$$
L_{c d}(t)=\langle\max (\theta(t))-\min (\theta(t))\rangle_{\text {region }}
$$

where $L_{c d}(t)$ is the maximum differences in the included angles $\theta(t)(0 \leq \theta(t) \leq \pi$ as in Fig. 2) with the positive $\mathrm{x}$-axis for the whole persons in the calculation region at time $t$. The closer $L_{c d}(t)$ gets to 0 , the smaller the local confusion degree of moving directions at cell $(i, j)$ is. The local moving risk $L_{r i s k}(t)$ at cell $(i, j)$ is calculated by Eq. 4 and Eq. 5:

$$
\begin{aligned}
& L_{r i s k}(t)=1-e^{-R(t)} \\
& R(t)=\frac{L_{d}(t) \cdot L_{c d}(t)}{L_{S}(t)}
\end{aligned}
$$

where $R(t)$ is the moving risk factor at cell $(i, j)$ for overall evaluating the above three parameters at time $t$. When $L_{d}(t), L_{c d}(t)$ increase and $L_{s}(t)$ decreases, the moving risk factor $R(t)$ gets larger, so is local moving risk $L_{r i s k}(t)$. The moving risk $M R_{i j}$ at cell $(i, j)$ during the whole moving time $T$ of one experiment is calculated by Eq. 6 :

$$
M R_{i j}=\sum_{m=1}^{N_{m}} \operatorname{Level}_{m}\left(L_{\text {risk }}(t)\right) \frac{\text { Duration }_{m}\left(L_{r i s k}(t)\right)}{T}
$$

where $N_{m}$ is the level number of the local moving risk $L_{\text {risk }}(t)$. $\operatorname{Level}_{m}\left(L_{\text {risk }}(t)\right)$ is the value corresponding to the level $m$ which $L_{\text {risk }}(t)$ belongs to. Normally $N_{m}$ equals to 4 , that means $L_{\text {risk }}(t)$ is divided to 4 levels, namely serious risk, relatively serious risk, general risk and low risk, while $\operatorname{Level}_{m}\left(L_{r i s k}(t)\right)$ is set to $4,3,2,1$, respectively. $\frac{\text { Duration }_{m}\left(L_{\text {risk }}(t)\right)}{T}$ measures the possibility that the moving risk at level $m$ appears.

\section{Results and Discussion}

\subsection{Channel Usage Analysis}

When the gaps $a$ between the barriers of the channelizing facilities are set to $0.7 \mathrm{~m}$ and $1.05 \mathrm{~m}, 6$ channels and 4 channels are formed in the entrance of the aisle, which are labeled as $1 \sim 6$ and $1 \sim 4$ from top to bottom as in Fig. 1) (a), respectively. Thus, the usage rates of the channels are analyzed to reveal how the participants entering from different sides of the waiting area choose the channels (Fig. 3)). The average usage rates of the 6 and 4 channels are 0.167 and 0.25 respectively as shown in the magenta dash lines in Fig. 3). 
First, when all participants enter from the waiting area (1), namely moving directly facing the entrance of the aisle, the middle channels obtain higher usage rates than the bilateral channels as shown in the red filled area. For 6 channels situation, the usage rates of channel 3 and 4 are $23.1 \%$ higher than the channel 2 and 5, while the usage rates of channel 2 and 5 are $69.6 \%$ higher than the channel 1 and 6 . For 4 channels situation, the usage rates of channel 2 and 3 are $11.9 \%$ higher than the channel 1 and 4 . Also, the inconsistency in the usage rates of the 6 channels is the largest (standard deviation: $S D=0.052$ ), comparing to other situations that participants from different waiting areas.

Second, when all participants enter from the waiting area (2), namely moving from one side towards the entrance of the aisle, the closer channels obtain higher usage rates than the farther channels as shown in the blue filled area. For 6 channels situation, the usage rates of channel $1 \sim 3$ are $17.2 \%$ higher than the channel $4 \sim 6$. For 4 channels situation, the usage rates of channel $1 \sim 2$ are $38.9 \%$ higher than the channel 3 4. Also, the inconsistency in the usage rates of the 4 channels is the largest (standard deviation: SD $=0.049$ ), comparing to other situations that participants from different waiting areas.

Third, when participants enter from the waiting area (2) and (3), namely moving from the two opposite sides towards the entrance of the aisle, the bilateral channels obtain higher usage rates than the middle channels as shown in the green filled area. For 6 channels situation, the usage rates of channel 1 and 6 are 16.9\% higher than the channel $2 \sim 5$. For 4 channels situation, the usage rates of channel 1 and 4 are $18.3 \%$ higher than the channel 2 and 3. Also, the inconsistency in the usage rates of the 6 channels is the smallest (standard deviation: $\mathrm{SD}=0.019$ ), comparing to other situations that participants from different waiting areas.

Fourth, when participants enter from the waiting area (1), (2) and (3), namely moving from three sides towards the entrance of the aisle, the channels closer to the waiting area (2) obtain higher usage rates than the channels closer to the waiting area (3) as shown in the yellow filled area, which means the participants who enter facing the entrance of the aisle might have a left-leaning behavior. For 6 channels situation, the usage rates of channel 1 3 are 10\% higher than the channel 4 6. For 4 channels situation, the usage rates of channel $1 \sim 2$ are $9.7 \%$ higher than the channel $3 \sim 4$. Also, the inconsistency in the usage rates of the 4 channels is the smallest (standard deviation: $\mathrm{SD}=0.013$ ), comparing to other situations that participants from different waiting areas.

Comparing the two setup methods of the channelizing facilities, when people can only enter from one side towards the entrance of the aisle, the inconsistency in the usage rates of the channels is inevitable. 6 channels situation is not suggested when people move facing the aisle (people tend to use the middle channels, which easily leads to conflicts), while 4 channels situation is not suggested when people move from the side of the aisle (people tend to use the closer channels, which also easily leads to conflicts). More entering sides will improve the inconsistency in the usage rates of the channels. 6 channels situation is more recommended when people move from the two opposite sides towards the entrance of the aisle, while 4 channels situation is more recommended when people move from three different sides of the aisle. 


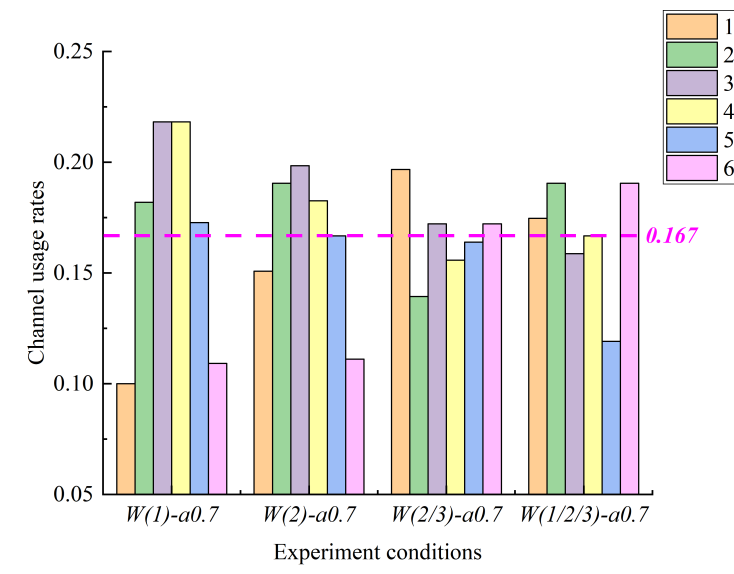

(a)

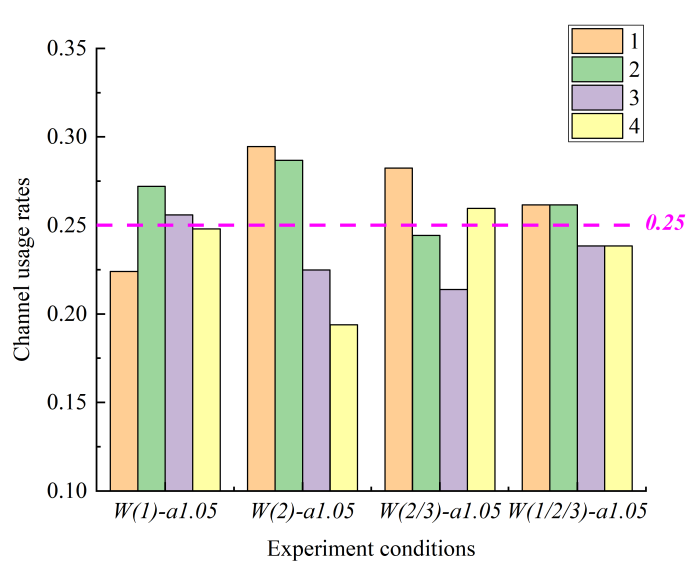

(b)

Figure 3 Radar map of the channel usage rates. (a) 6 channels, $a=0.7 m$. (b) 4 channels, $a=1.05 m$. The magenta dash lines represent the average usage rates of the 6 and 4 channels.

\subsection{Moving Risk Evaluation}

This section compares the instantaneous local density $L_{d}(t)$, local speed $L_{S}(t)$, local confusion degree of moving directions $L_{c d}(t)$ and local moving risk $L_{r i s k}(t)$, as well as the whole process of moving risk $M R$ within the confluence area in eight experiment conditions, according to the calculation methods in Sec. 3. The time interval is 1s. The duration of the whole process timed from the participants start to move till they finally pass through the channelizing facilities are 34s, 35s, 26s, 30s, 33s, 35s, 29s, 29s for the conditions (1)(8) respectively, so $L_{d}(t), L_{s}(t), L_{c d}(t), L_{\text {risk }}(t)$ at $15 \mathrm{~s}$ are analyzed in Tab. 2 to reflect a relatively stable entering process.

In general, since the color cloud charts of $L_{r i s k}(t)$ synthetically consider the influence of $L_{d}(t), L_{s}(t), L_{c d}(t)$ at the same time, they can reflect the distribution and magnitude of the high-risk zones in real time. In contrast to the single entering side conditions $(W(1))$, two or more entering sides conditions $(W(2 / 3)$ and $W(1 / 2 / 3))$ show broader scope of high-risk zones obviously, because the participants move slower and show more diversity in moving directions, as the lower $L_{s}(t)$ and higher $L_{c d}(t)$ shown in Tab. 2. In contrast to the narrower gaps conditions $(a=0.7 \mathrm{~m})$, the wider gaps conditions $(a=1.05 \mathrm{~m})$ show less high-risk zones relatively. The severe conflicts of the moving directions of the participants occur in front of the channelizing facilities are reduced $(a=1.05 m)$, so they can pass smoothly and gain higher moving speeds. For example $W(1 / 2 / 3)-a 0.7$ condition, a faster participant can be easily hindered by a slower person in front of him, especially when they prepare to pass through the one-person wide channelizing facilities, then he might change his direction and choose another channels. These phenomenons could lead great confusion of moving directions, even leading to the emergence of a very red area before the entrance of channels $\left(L_{c d}(t) \geq 2 / \pi\right)$ as shown in $W(1 / 2 / 3)-a 0.7$ condition.

Even so, $L_{\text {risk }}(t)$ only reflects the instantaneous moving risk within the confluence area, but the overall moving risk evaluation during the whole moving process lacks the con- 
sideration of the duration of the high-risk zones. To achieve this, $L_{\text {risk }}(t)$ (value range is 01 ) is divided to 4 levels: 00.25 belongs to level $1 ; 0.250 .5$ belongs to level $2 ; 0.50 .75$ belongs to level 3; 0.751 belongs to level 4. Higher level means higher risk. Then the duration of each level can be counted to calculating $M R$ using Eq. 6. According to the color cloud charts of $M R$ in Tab. 2, more risk-zones take on a strip shape (as white dotted lines marked in $M R$ charts) compared to the wider gaps conditions $(a=1.05 m)$, which could indicate the 'lane formation' effect appears clearer for the narrower gaps conditions $(a=0.7 \mathrm{~m})$. In addition, the end of the channelizing facilities, i.e. the topside and the bottom side in the color cloud chart, are always high moving risk areas, especially for the side entering conditions $(W(2), W(2 / 3)$ and $W(1 / 2 / 3))$. Because the participants entering from the side direction always tend to choose closer lanes, while the participants moving facing the aisle may have different walking tendency or choices to the left or the right (according to Sec. 4.1), the high risk is inevitable at end of the channelizing facilities. Yet surprisingly, two opposite entering sides conditions $W(2 / 3)$ show more severe and consecutive risk zones than the three entering sides conditions $W(1 / 2 / 3)$, which even occur in front of the middle channels. This can be explained by that more conflicts may appear when the participants entering from the side directions adopt the 'fastest path' strategy, instead of the 'shortest path' strategy, especially in $W(2 / 3)-a 0.7$ condition. These phenomenons are not existent in $W(1 / 2 / 3)$ conditions, because most participants moving from the waiting area (1) occupy the middle lanes and reduce the potential conflicts above. 


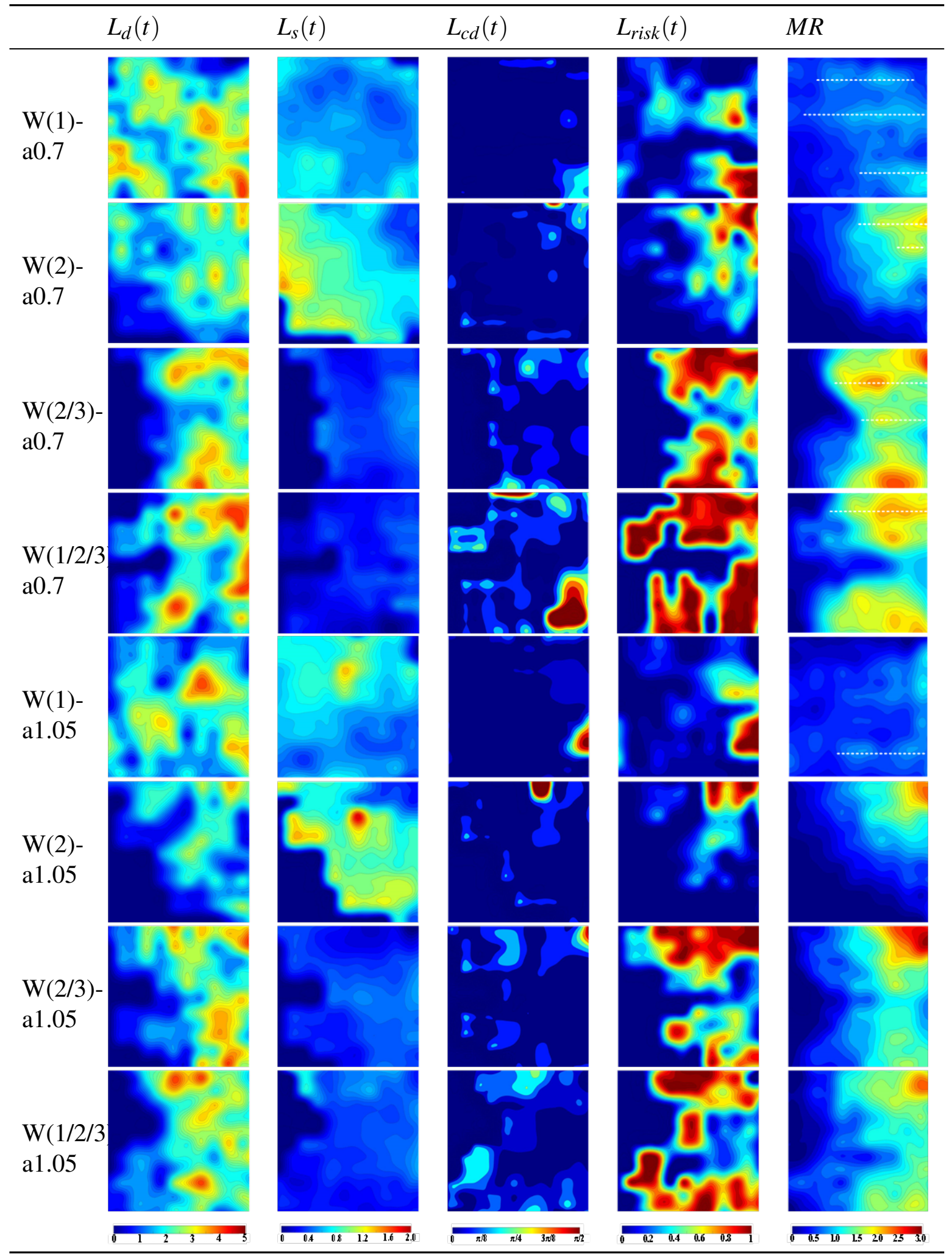

Table 2 Visual color cloud charts of $L_{d}(t), L_{s}(t), L_{c d}(t), L_{r i s k}(t)$ at 15 s and $M R$ of the whole process. 


\section{Conclusions}

This study investigates the moving risk of the crowds during the entering of the aisle in the presence of the channelizing barriers by proposing a risk visualization calculation method. This method allows to obtain a overall risk map of the the entrance confluence area and identify the formation of crowd organized patterns. The barrier setups are beneficial for balancing the channel usage rates, especially for multi-entry experiment conditions, but narrower gaps of barrier setups could lead to more consecutive and severer high-risk zones. The both ends of the channelizing barriers are higher moving risk zones for side-entry conditions, which needs to be closely focused on by the crowd managers. The results can efficiently identify hotspots and danger zones where crowds organize, which may help in the design of auxiliary crowd control facilities. More setting conditions of the channelizing facilities in front of the bottleneck are worth being considered and examined in our future research for safety crowd management.

Acknowledgements This research was supported by National Natural Science Foundation of China [grant No. 52072235], Shanghai Sailing Program [Grant No. 21YF1415800] and Humanity and Social Science foundation of Ministry of Education of China (Grant No. 20YJC630150). The authors deeply appreciate the supports.

\section{References}

[1] Seer, S., Bauer, D., Brandle, N., Ray, M.: Estimating pedestrian movement characteristics for crowd control at public transport facilities. In 2008 11th International IEEE Conference on Intelligent Transportation Systems, 742-747 (2008), doi:10.1109/itsc.2008.4732689

[2] Wang, J., Jin, B., Li, J., Chen, F., Wang, Z., Sun, J.: Method for guiding crowd evacuation at exit: The buffer zone. Safety science 118, 88-95 (2019), doi:10.1016/j.ssci.2019.05.014

[3] Zhuang, Y., Zhou, X. D., Ni, Y., Yang, L: Passengers' behavioral intentions towards congestion: Observational study of the entry restrictions at traffic bottleneck. KSCE Journal of Civil Engineering 21(6), 2393 (2017), doi:10.1007/s12205-016-1688-6

[4] Garcimartín, Á., Maza, D., Pastor, J. M., Parisi, D. R., Martín-Gómez, C., Zuriguel, I.: Redefining the role of obstacles in pedestrian evacuation. New Journal of Physics 20(12), 123025 (2018), doi:10.1088/1367-2630/aaf4ca

[5] Shi, X., Ye, Z., Shiwakoti, N., Tang, D., Lin, J.: Examining effect of architectural adjustment on pedestrian crowd flow at bottleneck. Physica A: Statistical Mechanics and its Applications 522, 350-364 (2019), doi:10.1016/j.physa.2019.01.086 
[6] Zhuang, Y., Liu, Z., Schadschneider, A., Yang, L., Huang, J.: Method for guiding crowd evacuation at exit: Exploring the behavior of self-organized queuing for pedestrian flow through a non-service bottleneck. Physica A: Statistical Mechanics and its Applications 562, 125186 (2021), doi:10.1016/j.physa.2020.125186

[7] Zhuang, Y., Liu, Z., Schadschneider, A., Fu, Z., Yang, L.: Experimental Analysis of the Restriction Mechanisms of Queuing on Pedestrian Flow at Bottleneck. In Traffic and Granular Flow 2019, 321-329 (2020), doi:10.1007/978-3-030-55973-1_40

[8] Feliciani, C., Nishinari, K.: Measurement of congestion and intrinsic risk in pedestrian crowds. Transportation research part C: emerging technologies 91, 124-155 (2018), doi:10.1016/j.trc.2018.03.027

[9] Lohner, R., Muhamad, B., Dambalmath, P., Haug, E.: Fundamental diagrams for specific very high density crowds. Collective Dynamics 2, 1-15 (2018), doi:10.17815/CD.2017.13

[10] Weidmann, U.: Transporttechnik der fußgänger: transporttechnische eigenschaften des fußgängerverkehrs, literaturauswertung. IVT Schriftenreihe 90, (1993), doi:10.3929/ethz-a-000687810

[11] Fruin, J. J.: Pedestrian planning and design 206, (1971)

[12] Helbing, D., Johansson, A., Al-Abideen, H. Z.: Dynamics of crowd disasters: An empirical study. Physical review E 75(4), 046109 (2007), doi:10.1103/PhysRevE.75.046109

[13] Alarifi, A., Al-Salman, A., Alsaleh, M., Alnafessah, A., Al-Hadhrami, S., Al-Ammar, M. A., Al-Khalifa, H. S.: Ultra wideband indoor positioning technologies: Analysis and recent advances. Sensors 16(5), 707 (2016), doi:10.3390/s16050707

[14] Maalek, R., Sadeghpour, F.: Accuracy assessment of ultra-wide band technology in locating dynamic resources in indoor scenarios. Automation in construction 63, 12-26 (2016), doi:10.1016/j.autcon.2015.11.009

[15] Bharadwaj, R., Swaisaenyakorn, S., Parini, C. G., Batchelor, J. C., Alomainy, A.: Impulse radio ultra-wideband communications for localization and tracking of human body and limbs movement for healthcare applications. IEEE Transactions on Antennas and Propagation 65(12), 7298-7309 (2017), doi:10.1109/TAP.2017.2759841 